\title{
Hirntrauma
}

\section{Zweifel am Nutzen der Druckmessung}

\author{
Bei einem schweren Schädelhirntrauma wird in der Regel auch der \\ Hirndruck überwacht. Diesen Aufwand kann man sich getrost sparen, \\ lautet das Fazit einer großen Studie. Doch nicht jeder traut den Daten.
}

$\mathrm{n}$ den meisten Industrieländern wird bei Patienten mit schwerem Schädelhirntrauma (SHT) eine Überwachung des intrakraniellen Drucks (ICP) empfohlen. Der Nutzen liegt auf der Hand: Steigt der Druck über $25 \mathrm{mmHg}$, erhöht sich das Sterberisiko drastisch. Ärzte können also schnell eingreifen, wenn der Hirndruckmonitor Alarm schlägt. Ob die Drucküberwachung mittels Katheter letztlich zu einem besseren Behandlungsergebnis führt als eine Therapie, die auf einer klinischen Beurteilung des Druckrisikos und der Bildgebung beruht - das konnte bislang allerdings noch nicht klar belegt werden.

\section{Widersprüchliche Studiendaten}

Die Empfehlungen zum Hirndruckmonitoring gründen sich lediglich auf Beobachtungsdaten, nicht aber auf evidenzbasierten Studien, sagte Privatdozentin Asita Sarrafzadeh von der Universität Genf bei der Arbeitstagung Neurologische Intensivmedizin (ANIM) in Mannheim. Und da, wo es Studien gebe, hätten sie nicht immer Vorteile für die ICP-Messung oder das Monitoring des zerebralen Perfusionsdrucks (CPP) erbracht. Die Neurologin nannte etwa eine niederländische Kohortenstudie aus dem Jahr 2005: Hier wurden die Ergebnisse von zwei Traumazentren verglichen. In dem einen erfolgte die Behandlung von Patienten mit SHT anhand der ICP/CPP-Kontrolle, im anderen ausschließlich über klinische Symptome sowie Zeichen eines erhöhten Hirndrucks in der Bildgebung. Beim Behandlungserfolg gab es jedoch keinen Unterschied zwischen den beiden Zentren - Mortalität und Funktionsniveau der Überlebenden waren vergleichbar. Unterschiede gab es lediglich bei Therapiekosten, -dauer und -intensität: Die
ICP-basierte Therapie war aufwändiger, teurer und verbrauchte mehr Ressourcen, ohne dass die Patienten messbar davon profitierten [1].

$\mathrm{Zu}$ anderen Ergebnissen kam eine US-Studie, bei der prospektive Registerdaten ausgewertet wurden: Hier waren die Überlebenschancen für Patienten mit ICP-basierter Therapie nach zwei Wochen signifikant höher als bei Patienten, deren Hirndruck nicht überwacht wurde.

\section{Erste prospektive randomisierte Studie}

Klarheit sollte nun die bisher einzige prospektive randomisierte und kontrollierte Studie schaffen, deren Ergebnisse kürzlich veröffentlicht wurden [2]. Da eine solche Studie aufgrund der aktuellen Empfehlungen in Industrieländern ethisch kaum machbar erschien, wählten die Studienautoren sechs Zentren in Bolivien und Ecuador - in diesen Ländern ist das ICP-Monitoring bislang eher unüblich. Die Zentren waren alle modern ausgerüstet, verfügten über einen 24-Stunden-Zugang zu einem CT und zu einer Hirnchirurgie-Abteilung. Alle Patienten hatten schwere SHT, der Wert auf der GCS ("Glasgow Coma Scale“, 3-15 Punkte) lag zwischen 3 und 8 Punkten, beim niedrigsten Wert von 3 Punkten mussten die Ärzte aber noch Überlebenschancen erkennen.

Insgesamt behandelten die Ärzte nun 324 Patienten entweder ICP-basiert oder aufgrund klinischer Zeichen und Bildgebung. Das Ergebnis: Weder bei der Sterberate $39 \%$ in der ICP-Gruppe, $40 \%$ in der Kontrollgruppe) noch beim Funktionsniveau (63\% vs. $60 \%$ mit gutem klinischem Ergebnis) gab es signifikante Unterschiede. Der einzige Vorteil ließ sich bei Kosten und Aufwand erkennen: Hier schnitt die ICP-Gruppe im Gegensatz zur niederländischen Studie besser $a b$ - es wurden seltener drucksenkende Maßnahmen eingeleitet als in der Kontrollgruppe.

Ein Problem der Studie zeigte sich bei der hohen Mortalitätsrate von $40 \%$. Sie ließ sich damit begründen, dass die Patienten in den Studienländern nicht immer zeitnah in die Klinik kamen und statt im Notarztwagen oft von Angehörigen im Auto oder Taxi ins Krankenhaus gebracht wurden - dies könnte das Ergebnis verzerrt haben. Ein Teilnehmer der ANIM-Tagung bemängelte zudem, dass in der ICP-Gruppe nur bei $7 \%$ der Patienten während des Monitorings ein unerwarteter Druckanstieg beobachtet wurde, sodass letztlich die Patienten in beiden Gruppen kaum unterschiedlich behandelt wurden.

\section{Widersprüchliche Studiendaten}

Kritik gab es auch von dem Neurochirurgen Professor Peter Le Roux aus Philadelphia/USA, der die evidenzbasierte Medizin auf der Intensivstation generell infrage stellte. „Wir treffen hier Entscheidungen aufgrund universeller Prinzipien und praktischer Erkenntnisse. Sie sind genau auf einen bestimmten Patienten zugeschnitten. Bei Studien besteht jedoch die Gefahr, dass wir solche Patienten der falschen Therapiegruppe zuordnen müssen." Le Roux erinnerte auch daran, dass das Monitoring zunächst nur Daten liefert, es komme aber darauf an, was man mit den Daten mache. Beim Hirndruck infolge eines SHT sei das Problem, dass man meist nicht wisse, was genau den Druckanstieg auslöse. Das Management sei daher mit oder ohne ICP-Messung sehr ähnlich.

Thomas Müller

1. Cremer OL et al. Crit Care Med 2005; 33: 2207-13

2. Chesnut RM et al. N Engl J Med 2012; 367: 2471-81

Symposium „Management of Traumatic Brain Injury (TBI)", ANIM, Mannheim, 24.1.2013 